\title{
Emotional Expression and Its Applications
}

\author{
Ho Seok Ahn · Dong-Wook Lee
}

Published online: 27 September 2013

(C) Springer Science+Business Media Dordrecht 2013

Humans are social animals that require close relationships with other people. Moreover, humans have relationships with pets in our society, and some robots are regarded as pets or companions, for example, AIBO and PARO. It means that robots can be social beings in human society, and we call these kinds of robots as social robots.

Social robots communicate with humans as their partners in our daily life environment, and Human-Robot Interaction is one of the core technologies for social robots. Emotional expressions and interactions are essential to social robots and applied to various areas, for example, performing in theater, assisting humans in home, and healing humans in hospital, etc.

The contributions in this special issue are on the emotional expression and applications of social robots, covering the topics of facial expression, recognizing and imitating human emotions, emotional postures, emotion-based humanrobot interaction, as well as social effects of emotional expressions and interaction.

The first paper is on "Visualization of Facial Expression Deformation Applied to the Mechanism Improvement of Face Robot" by Chyi-Yeu Lin, Li-Chieh Cheng and ChunChia Huang. The authors present a design approach, which uses reverse engineering techniques of three dimensional measurement and analysis, to visualize some critical facial

H.S. Ahn ( $\square)$

Robotics group, Department of Electrical and Computer Engineering, University of Auckland, Science Building 301, 38 Princess Street, Auckland, New Zealand e-mail: hoseoka@gmail.com

D.-W. Lee

KITECH, 1271-18, Sa 3-dong, Sangrok-gu, Ansan-si, Gyeonggi-do, Republic of Korea

e-mail: dwlee@kitech.re.kr motion data, including facial skin localized deformations, motion directions of facial features, and displacements of facial skin elements on a human face in different facial expressional states.

The second paper, titled "Emotional Postures for the Humanoid-Robot Nao", and authored by Mustafa Suphi Erden, presents the development of emotional postures for the humanoid robot Nao. The approach is based on adaptation of the postures that are developed for a virtual human body model to the case of the physical robot Nao.

The third paper, "Increasing Helpfulness towards a Robot by Emotional Adaption to the User", authored by Barbara Kühnlenz, Stefan Sosnowski, Malte Buß, Dirk Wollherr, Kolja Kühnlenz and Martin Buss describes an emotional adaption approach to proactively trigger increased helpfulness towards a robot in task-related human-robot interaction. This approach aims at inducing empathy, paired with a feeling of similarity in human users towards the robot based on social-psychological predictions of human behaviour.

The fourth paper by Ana Lucia Pais, Brenna Dee Argall and Aude Billard is on the "Assessing Interaction Dynamics in the Context of Robot Programming by Demonstration". The authors focus on human-robot interaction particularities that occur during programming by demonstration using the iCub robot that shows facially-displayed expressions. The authors determine the preferred and more effective way of providing feedback in relation to the robot's tactile sensing, in order to improve the teaching interaction and to keep the users engaged throughout the interaction.

In the fifth paper, on "Effects of Eye Contact and Iconic Gestures on Message Retention in Human-Robot Interaction", by Elena Torta, Elisabeth van Dijk and Raymond H. Cuijpers, the effects of iconic gestures and eye contact on message retention in human-robot interaction are investigated in a series of experiments. A humanoid robot gives 
short verbal messages to participants, accompanied either by iconic gestures or no gestures while making eye contact with the participant or looking away.

In the sixth paper, titled "Imitating Human Emotions with Artificial Facial Expressions", by David O Johnson, Raymond H Cuijpers and David van der Pol, the method for imitating human emotion is addressed. The authors investigate how LED patterns around the eyes of Aldebaran's Nao robot by performing two experiments; they examine the LED color, intensity, frequency, sharpness, and orientation that humans associate with different emotions in the first experiment, and measure how well humans recognize the created LED patterns as the emotions in the second experiment.

The seventh article, on "Cross-Cultural Perspectives on Emotion Expressive Humanoid Robotic Head: Recognition of Facial Expressions and Symbols", is authored by Gabriele Trovato, Tatsuhiro Kishi, Nobutsuna Endo, Massimiliano Zecca, Kenji Hashimoto and Atsuo Takanishi. They introduce a generation system that produces facial expressions, and apply it to the 24 degrees of freedom head of the humanoid social robot KOBIAN-R for adapting to different cultural backgrounds.

The eighth article by Gentiane Venture and Ritta Baddoura is on the "Social vs. Useful HRI: experiencing the familiar, perceiving the robot as a sociable partner and responding to its actions". The authors explore the human affective state of the familiar during a new or unknown situation as it relates to interacting with a robot. They analyse the participants' reactions to the robot's actions, the motion of their arms, and their answers to some parts of a questionnaire designed to measure their experience of the familiar and the robot's sociability.

In the ninth paper, titled "Human-like Interaction Skills for the Mobile Communication Robot Robotinho", and authored by Matthias Nieuwenhuisen and Sven Behnke, presents the anthropomorphic mobile communication robot Robotinho includes an expressive communication head to display emotions and multimodal dialog system incorporates gestures, facial expression, body language, and speech. They describe the behaviors developed for interaction with inexperienced users in a museum tour guide scenario.

The tenth article, on "Teleoperated android robot as emotion regulation media", is authored by Shuichi Nishio, Koichi Taura, Hidenobu Sumioka and Hiroshi Ishiguro. They experimentally examine whether changes in the facial expressions of teleoperated androids can affect and regulate operators' emotion, based on the facial feedback theory of emotion and the phenomenon of body ownership transfer to the robot.

The eleventh paper is entitled "Design and Technologies for Understanding Older Adults Social Interactions in Retirement Communities" by claudia b rebola, Gbolabo Ogunmakin and Patricio A Vela. The purpose of this paper is to describe the design, development, and technology studies conducted for understanding the social interactions of older adults in retirement communities. This study is relevant for the field of social robotics as an as example of a realistic application domain.

The twelfth paper by John-John Cabibihan, Hifza Javed, Marcelo Ang Jr. and Sharifah Mariam Aljunied entitled "Why robots? A survey on the roles and benefits of social robots in the therapy of children with autism" reviews the use of socially interactive robots to assist in the therapy of children with autism. Especially, experimental data from the surveyed works are extracted and analysed in terms of the target behaviours and how each robot is used during a therapy session to achieve these behaviours.

The thirteenth paper is on "Representing Affective Facial Expressions for Robots and Embodied Conversational Agents by Facial Landmarks" by Caixia Liu, Jaap Ham, Eric Postma, Cees Midden, Bart Joosten and Martijn Goudbeek. The goal of this paper is to investigate whether digitallyextracted facial landmarks contain sufficient information to enable the facial expressions to be recognized by humans.

The final paper, on "Effects of $3 D$ Shape and Texture on Gender Identification for a Retro-Projected Face Screen", authored by Takaaki Kuratate, Marcia Riley and Gordon Cheng, presents retro-projected face display robot that is able to show a variety of faces varying in both realism and individual appearance. Additionally, the authors present three cases of morphed images: high quality texture, low quality texture, and averaged face texture from low quality data.

We would like to thank Editor in Chief Shuzhi Sam Ge and Assistant Editor John-John Cabibihan from the Editorial Board, as well as Anand David and Nathalie Jacobs from Springer for their guidance and advice.

We very warmly want to thank our reviewers who set the high standards of this special issue: Jordi Vallverdu, Joon-Yong Lee, Laura Boccanfuso, Rosario Sorbello, Luca Brayda, Astrid Marieke Rosenthal-von der Pütten, Jorge Solis, Jaap Ham, Thomas Holz, David O Johnson, Alan Richard Wagner, Tatsuya Nomura, Gentiane Venture, John Zhong Zhang, Anand Panangadan, Dilip Kumar Limbu, Joost Broekens, Selma Sabanovic, I-Han Kuo, Joelle Pineau, Bram Vanderborght, Manuel Giuliani, Hirotaka Osawa, Nathan Kirchner, Alexandra Kirsch, Emrah Akin Sisbot, Karolina Zawieska, Cetin Mericli, Miguel A. Salichs, Marco Ceccarelli, Ryad Chellali.

Guest editors

Ho Seok Ahn is a postdoctoral research fellow at Robotics research group, Department of Electrical and Computer Engineering, University of Auckland, New Zealand. He received his Ph.D. degree from Seoul National University, Republic of Korea. He was a research scientist at Advanced Telecommunications Research Institute International (ATR), Japan, a senior researcher at Korea Institute of Indus- 
trial Technology (KITECH), Republic of Korea, a lecture at University of Science \& Technology (UST), Republic of Korea, and a visiting researcher at Advanced Industrial Science and Technology (AIST), Japan. His research interests include social robots, cultural robots, facial robots, artificial emotional systems, Human-Robot Interaction, healthcare robots, android robots, humanoid robots, intelligent service robots, and modular robot systems.

Dong-Wook Lee is a principal researcher at Korea Institute of Industrial Technology (KITECH) and an associate professor at University of
Science \& Technology (UST), Republic of Korea, since 2005. He received his B.S., M.S. and Ph.D. degrees from Chung-Ang University, Republic of Korea, in 1996, 1998, and 2000, respectively. He was a research professor at Chung-Ang University Republic of Korea, from 2002 to 2004 . He was a post-doctoral researcher at the University of Tennessee, USA, from 2004 to 2005 . His research interests include android robots, cognitive \& affective Human-Robot Interaction, artificial life, and evolutionary computation. 